\title{
Information Fusion as Knowledge Extraction in an Information Processing System
}

\author{
Arwin Datumaya Wahyudi Sumari
}

\begin{abstract}
Knowledge extraction is a means of obtaining knowledge from a phenomenon occured in the environment. Knowledge extraction as part of a process within an information processing system is a very easy task for human brain to do. In this paper we present a new technique for knowledge extraction called information fusion. In the creation of new knowledge, the brain does inferencing to obtain an inference. In essence, when doping the inferencing, the brain is fusing information obtained from the environment with the knowledge already stored in it. The obtained inference is new knowledge of the system while the occured mechanism is called as knowledge extraction. We have developed a method called A3S (Arwin-Adang-Aciek-Sembiring) for knowlegde extraction that was already applied to our system called KnowledgeGrowing System (KGS). We also present an example with real-life data to show the work of A3S in performing knowledge extraction
\end{abstract}

Keywords-A3S, Artificial Intelligence, information fusion, knowledge extraction, $K G S$

\section{Introduction}

Processing information can be done very easily by human brain. Any kind of information including image, text, voice and taste that come from human sensory organ can be processed in just very very small time. The output of the process or the processed information, can be used as a basis for decision making or for the next processes. In order to make this explanation understandable, we can refer to ourself. The decision we made is always based on the information from the environment which is sensed by our sensory organs. The sensed phenomenon or information, then is delivered to our brain to be processed to obtain knowledge. The knowledge is comprehensive information that describes the sensed phenomenon. By knowing the phenomenon clearly, you can make a proper decision to anticipate what may occur at that time or in the future. The mechanism occured within human brain from the start, namely sense the phenomenon occurs in the enviroment, do inference to the information regarding to the sensed phenomenon and continued with formulate the decision, make a decision and act to it, is simply represented by SIDA (Sense-Inference-Decide and Act) cycle in Fig. 1 which models the human thought.

\section{Arwin Datumaya Wahyudi Sumari}

Cognitive Artificial Intelligence Research Group (CAIRG)

School of Electrical Engineering and Informatics

Institut Teknologi Bandung, Indonesia

\section{Adang Suwandi Ahmad}

Cognitive Artificial Intelligence Research Group (CAIRG)

School of Electrical Engineering and Informatics

Institut Teknologi Bandung opIndonesia

\author{
Adang Suwandi Ahmad
}

This is a continuous cycle and will never stop. Why? As long as human lives, his information processing system will always work to process the information delivered from his sensory organs. If we take a look deeper on the second phase of the SIDA cycle namely Inference and decision formulation, we can see clearly that the information delivered from the sensory organs will be inferenced to obtain inference, that is a guess of the sensed phenomenon. We also have known that the human sensory organs consist of five elements namely, eyes, ears, nose, skin and tongue. The brain can have information from only one sensor. But in most cases, in order to have more complete information regarding the sensed phenomenon, information from more than one sensors is more prioritized to ensure its validity. The information from more than one sensor firstly has to be fused before inferenced in the next process.

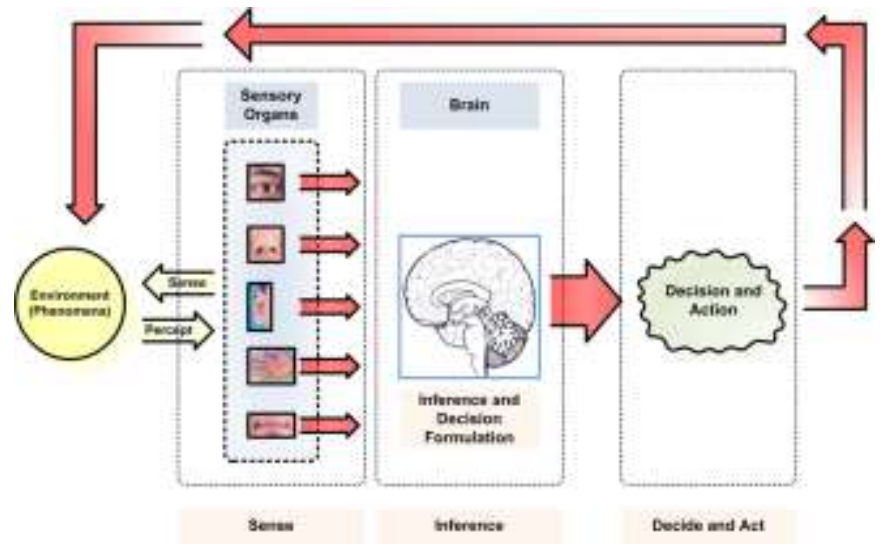

Fig. 1 SIDA cycle of human thought model [1].

The process to obtain inference can also be said as inferring or inferencing but they are different. The different between the two words is not only in their definition but also on the usage of them. Inferring is defined as to derive by reasoning; conclude or judge from premises or evidences. On the other hand, inferencing is the practice of inferring the meaning of an unfamiliar word or expression from the meaning of familiar words occurring with it in a context together with one's knowledge of or beliefs about the world. Inferring just needs premises or evidences to obtain an inference but inferencing is more complicated because it requires knowledge to obtain an inference. Even though those terminologies come from psycholinguistic field, word "inferencing" has wider application other fields such as Artificial Intelligence (AI) field [2]. Inference as the guess of the sensed phenomenon will become brain's new knowledge regarding such phenomenon and it will be used to guess the future phenomenon sensed by the sensory organs. The process of obtaining an inference to become new knowledge is called as knowledge extraction. 
In order to give a concise explanation on our concept and the application of the concept, the delivery in this paper will be as follow. A short introduction regarding the essence of the paper has been given in Section I. Some essential theoretical background will be given in Section II, followed by Section III where we will show you the development of the method for knowledge extraction. An example on the method application to give a solution to a real-life problem will be given in Section IV. The last part is Section $\mathrm{V}$ where we give some remarks regarding the method as well as the next step of our research.

\section{Theoretical Background}

\section{A. Models of Information Processing System}

Essentially, information processing model is a theory of human development that uses the computer as a metaphor for explaining thought processes. Similar to computers, humans transform information to solve cognitive problems. Development is viewed in terms of changes in memorystorage capacities and use of different types of cognitive strategies [3]. On the other hand, information processing can be defined as the acquisition, recording, organization, retrieval, display, and dissemination of information [4]. In this section we only take a look on three information processing models, namely Wicken's model, Welford's model, and Whiting's model.

- Wickens' Model. Wicken models the human information processing by dividing it into seven parts namely, sensory processing, short-term sensory store, perceptual encoding, decision making and response selection, response execution, feedback and information flow and attention. Wicken's model is used for giving an introduction of some of the basics of cognitive science that apply to Human Factors design, which in this case is given to people who work in aviation industry.

- Welford's Model. Another better known model is Welford's information processing model [6]. This model has three processes namely stimulus identification stage, response identification/ selection stage, and response programming stage. The elements of the model are sensory input, short and long term memories, decision process and action. The decision process is represented by a process named translation from perception to action which is a selection the most appropriate response from alternatives as the resulted of information processing. After the decision is made, the effector control controls which effector will do the response in term of action. This action is done to the phenomenon in the environment which is reperesented by external object..

- Whiting's Model. Whiting's model of information processing introduced in 1969 as comparison to Welford's model. This model has seven elements [7] with some relevan modifications namely, things, input data, translatory mechanism, effector mechanism, output dan feedback data. The most important matter in this model is the inputs from perceptual mechanism are directly processed by tran.slatory mechanism to become knowledge to be delivered to effector mechanism

\section{B. Knowledge Extraction}

There are many definitions on what is called knowledge extraction. One defines knowledge extraction as the non-trivial process of identifying valid, novel, potentially useful and ultimately understandable patterns from large data collections. Other literature says that knowledge extraction is the creation of knowledge from structured (relational databases, eXtensible Markup Language/XML) and unstructured (text, documents, images) sources. From the two definitions, we can extract the essences that knowledge extraction is a process, the creation of knowledge and carried out by information processing system.

Most of works in knowledge extraction are for getting knowledge from text documents such as works done by [8-12]. They used different methods to do knowledge extraction such as Fuzzy techniques, Support Vector Machine (SVM), decision tree, clustering techniques and Naive Bayes. At the writing of this paper, it is very hard to find a literature regarding knowledge extraction that emulate how the human brain works on this mechanism.

\section{Information Fusion}

The information fusion originates from the examination of how human can make a decision or an action in accurate and quick manner after having much information regarding a certain situation. Besides that, human can also predict or estimate the situation that is probably occured in the future by combining recent information with his/her previous knowledge. Human obtains much information from his/her sensing organs which comprises eyes, ears, nose, skin, and tongue. On the other hand, human also gains information from other information multi-source such as making communications with other people. After gathering information for multi-source, the brain does its job by fusing the information to become comprehensive information as the basis for decision making. This mechanism is called as human information fusion system and it is done continuously in human everyday life.

Inferencing is the practice of inferring the meaning of an unfamiliar word or expression from the meaning of familiar words occurring with it in a context together with one's knowledge of or beliefs about the world. Given a word or we can say a phenomenon. The word can be given in form of voice or writing on the blackboard or given in those two forms simultaneously. As we have agreed that information which is delivered from multi-source is prioritized, then the brain will only process simultant information from ear and eyes. The inferencing will be carried out by fusing the information from 
multi-source (ear and eyes) and prior knowledge already stored in brain's memory. During the inferencing, brain will carry out information fusion and the number of fusion cycles is depended on the number of SIDA cycle loops. If it is only one-time information delivery then the brain will obtain an inference as the representation of comprehensive information resulted from information fusion. This inference is called as new knowledge of the brain reagarding the sensed phenomenon. The mechanism of having new knowledge is called as knowledge extraction.

\section{Developing the Method for Knowledge Extraction}

Essentially, Knowledge-Growing System (KGS) is a system that is capable of growing its knowledge along with the accretion of information as the time passes. On the other hand, knowledge extraction is the creation of knowledge. Meaning that there will be new knowledge after doing knowledge extraction. The only different is in KGS the knowledge of phenomenon is grown from nothing, while in knowledge extraction the new knowledge is obtained from patterns in a phenomenon. But in essence they do the same thing, namely obtain new knowledge. We have built new method for growing the knowledge, which can be said as knowledge extraction in KGS, called A3S (Arwin-Adang-AciekSembiring)[13]. On the next texts we will deliver the development of our method for knowledge extraction.

\section{A. SIDA Cycle Revisited}

Take a look back to Fig. 1 that depicts SIDA human thought cycle that has three stages with explanations as follow:

- Sense. This phase is represented by the activities of the sensory organs to sense and to perceive the environment (phenomenon) dynamics.

- Inference and Decision Formulation. In this phase, inference or new knowledge is grown through fusion or combination of information delivered from Sense phase with the existing knowledge.

- Decide\&Act. This phase is where the product of the previous phase is implemented to affect the environment.

\section{B. Knowlegde-Growing Mechanism as a Method for Knowledge Extraction}

Inference and Decision Formulation is the phase where knowledge-growing mechanism occurs through fusion or combination of new information with the existing knowledge in order to obtaining inference or new knowledge. The illustration of the knowledge-growing mechanism. Multisensor terminology used in the table represents the five sensory organs belonged to human. This terminology can be extended to any system with more than five sensory apparatus.
TABLE I. THE ILLUSTRATION OF THE KNOWLEDGE-GROWING MECHANISM [14]

\begin{tabular}{|c|c|c|c|c|c|c|c|c|}
\hline \multirow{2}{*}{ th Sensor } & \multicolumn{8}{|c|}{$j^{\text {th }}$ Fused Information from Multi-Sensor } \\
\hline & 1 & 2 & 3 & +2 & $J$ & $\ldots$ & $\ldots$ & $\lambda$ \\
\hline 1 & 1 & $P\left(v_{2}^{2}\right)$ & $P\left(u_{1}^{3}\right)$ & $\ldots$ & $P\left(u_{1}^{2}\right)$ & $\ldots$ & $\ldots$ & $P\left(v_{2}^{3}\right)$ \\
\hline 2 & $\left.P\left(y_{1}^{2}\right)\right\}$ & 0 & $\overline{0}$ & 4 & $\overline{0}$ & $\ldots$ & $\ldots$ & $P\left(v_{1}^{2}\right)$ \\
\hline$\cdots$ & $\ldots !$ & + & $\cdots$ & $+\cdots$ & +4 & + & $\ldots$ & $\ldots$ \\
\hline t & $\overline{0}$ & 0 & $P\left(u_{3}\right)$ & $\ldots$ & $P\left(u^{\prime}\right)$ & $\ldots$ & $\ldots$ & $P\left(w_{j}^{j}\right)$ \\
\hline$\ldots$ & $\ldots$ & $\ldots$ & $\ldots$ & $\ldots$ & $\ldots$ & $\ldots$ & $\ldots$ & $\ldots$ \\
\hline 8 & 0 & 0 & 0 & 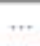 & 0 & $\ldots$ & $\ldots$ & $P\left(v_{1}^{3}\right)$ \\
\hline$\Psi$ & & $P\left(\psi_{1}^{2}\right.$ & $\boldsymbol{P}\left(\boldsymbol{\psi}_{1}^{3}\right)$ & $\cdots$ & $\boldsymbol{P}\left(\boldsymbol{\psi}_{1}^{\gamma}\right)$ & $\ldots$ & $\ldots$ & $\boldsymbol{P}\left(\boldsymbol{\psi}_{1}^{\alpha}\right)$ \\
\hline
\end{tabular}

Let us assume that $\delta=1, \ldots, i, \ldots, n$ is the number of sensor or information source of information multi-source or multi-sensor, $\lambda=1, \ldots, j, \ldots, m$ is a collection of hypotheses or multi-hypothesis of phenomenon regarding the information supplied by the multi-sensor. At the end of the computation, $\lambda$ is also functioned as the numbers of fused information from multi-sensor that explain a collection of individual phenomenon based on the multi-hypothesis.

Notation $P\left(v_{j}^{i}\right)$ represents the probability hypothesis $j$ is true given information sensed and perceived by sensor $i$. The Degree of Certainty (DoC) [18] represented by $P\left(\psi_{1}^{j}\right)$ defines that hypothesis $j$ is selected based on the fusion of the information delivered from multi-sensor, that is, from $P\left(v_{j}^{1}\right)$ to $P\left(v_{\delta}^{j}\right)$ where $j=1, \ldots, \lambda$. The subscript " 1 " in notation $P\left(\psi_{1}^{j}\right)$ means that the computation results are DoC at time 1 or the first observation time. This number is required if we want to have the next observation to be computed. The information fusion to obtain a collection of $\mathrm{DoC}$ is given in (1).

$$
P\left(\psi_{1}^{j}\right)=\frac{\sum_{i=1}^{\delta} P\left(v_{j}^{i}\right)}{\delta}
$$

where $P\left(\psi_{1}^{j}\right) \in \Psi$ and it is called as New Knowledge Probability Distribution (NKPD) [1][13]. This is a collection of information that can be furthered extracted to obtain inference or new knowledge. The inference or new knowledge at this point can be obtained by applying (2).

$$
P\left(\psi_{1}^{j}\right)_{\text {estimate }}=\square\left[P\left(\psi_{1}^{j}\right)\right]
$$

where $\square[\ldots]=\max [\ldots]$. This is a new notation proposed as a part of $\mathrm{A} 3 \mathrm{~S}$ information-inferencing fusion method which is the basis for KGS knowledge-growing mechanism [13]. 
$P\left(\psi_{1}^{j}\right)_{\text {estimate }}$ is the inference of $P\left(\psi_{1}^{j}\right) \in \Psi$ which later become new knowledge of KGS. The growing of knowledge over time is obtained by replace the first column of Table I to time parameter. The advancement of A3S method that already involves time parameter gave rise to new method called Observation Multi-time A3S (OMA3S) method and knowledge distribution resulted from the application of this method is called as New Knowledge Probability Distribution over Time (NKPDT) [14].

Back to Table I, the mechanism occurred in the table illustrates that the brain tries to guess or recognize the observed phenomenon or external object by collecting information through its sensory organs (multi-sensor). Actually in the beginning it knows nothing about the phenomenon. Therefore, when it uses different sensor, it perceives the same phenomenon as different one. By using information from several sensors simultaneously, it will have more complete knowledge regarding the phenomenon. The knowledge acquisition is carried out on some time interval using OMA3S in order to ensure that it has sufficient knowledge to recognize the phenomenon. The quality of the grown knowledge is measured using (3).

$$
\text { DoC }=\left|P\left(\theta_{j}\right)_{\text {estimate }}-\phi_{1}^{j}\right| \times 100 \%
$$

with $P\left(\theta_{j}\right)$ is information-inferencing of $P\left(\psi_{\gamma}^{j}\right)$ after several observation time to the phenomenon and $\phi_{1}^{j}$ is the knowledge probability of the most correct fused-information $j$ which represents the phenomenon at observation time $\gamma_{1}$. In the case of knowledge extraction, (3) can be used to assess how good is the extracted knowledge compared to the true phenomenon being observed.

\section{Iv. The Application of the Knowledge Extraction Method - A3S Method}

In this section we will deliver an example of the use of A3S method for extracting new knowledge from patterns of phenomema. We did this research in one of Indonesian national body and developed an intelligent information fusion system for decision support which is tasked to obtain new knowledge after given a series of pattern from a sensed phenomenon. Refer to Tabel I and (1), the system has 42 indications as the generalization of sensors and 8 hypotheses as the generalization of possible answer of the sensed phenomenon.

As the example, we only use 10 indications as the inputs to the system and then are given to the system. Fig. 2 shows the results of NKPD for the first computation and this result can be saved to be used for the next computation. The new knowledge from the first observation can be exracted by applying (2) as follow.

$$
\begin{aligned}
P\left(\psi_{1}^{j}\right)_{\text {estimate }} & =\square\left[P\left(\psi_{1}^{j}\right)\right] \\
& =\square[0.15,0.22,0.09,0.13,0.24,0.21,0.13,0.08] \\
& =0.24
\end{aligned}
$$

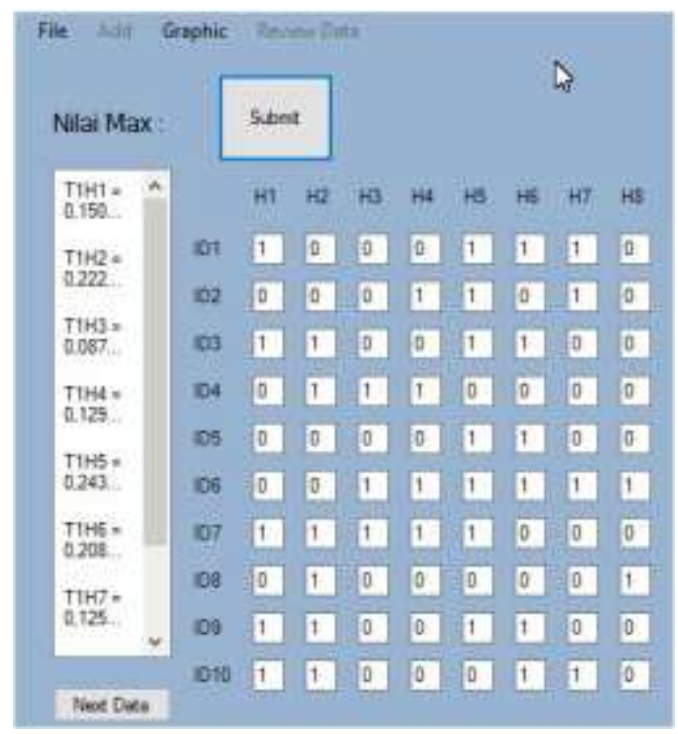

Fig. 2 The output of the system. The NKPD is shown at the left side with variable named $T_{i} H_{j}=P\left(\psi_{1}^{j}\right)$.

The result shows that the $P\left(\psi_{1}^{5}\right)$ has the highest probability, meaning that $H_{5}$ is the extracted knowledge from a pattern of $0 \mathrm{~s}$ and $1 \mathrm{~s}$ of the sensed phenomenon given to the system. The process of obtaining new knowledge does not stop at the first observation. We did the second and third observation with the results in graphic form as depicted in Fig. 3.

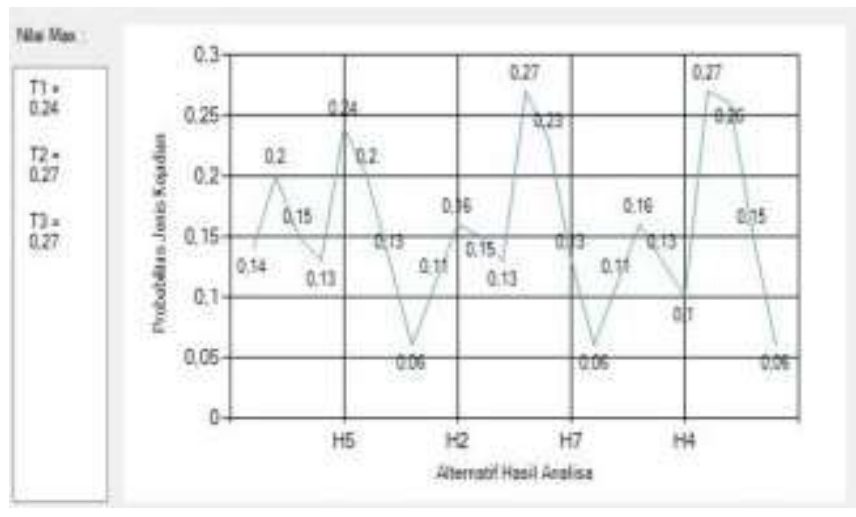

Fig. 3 The computation result from the second and third observation.

The summary of all observation is given in Tabel II. Because for the next computation we involve time as the measurement of the observation, so there is a slight change for the row variable. 
TABLE II. CONFIGURATION OF TIME AND HyPOTHESIS

\begin{tabular}{|c|c|c|c|c|c|c|c|c|}
\hline \multirow{2}{*}{$\begin{array}{c}-\mathbf{t} \\
\text { indica- } \\
\text { tion }\end{array}$} & \multicolumn{7}{|c|}{$-\mathbf{j}$ hypothesis } \\
\cline { 2 - 9 } & $H_{1}$ & $H_{2}$ & $H_{3}$ & $H_{4}$ & $H_{5}$ & $H_{6}$ & $H_{7}$ & $H_{8}$ \\
\hline$t_{1}$ & 0.14 & 0.2 & 0.15 & 0.13 & 0.24 & 0.2 & 0.13 & 0.06 \\
\hline$t_{2}$ & 0.11 & 0.16 & 0.15 & 0.13 & 0.27 & 0.23 & 0.13 & 0.06 \\
\hline$t_{3}$ & 0.11 & 0.16 & 0.15 & 0.1 & 0.27 & 0.26 & 0.15 & 0.06 \\
\hline
\end{tabular}

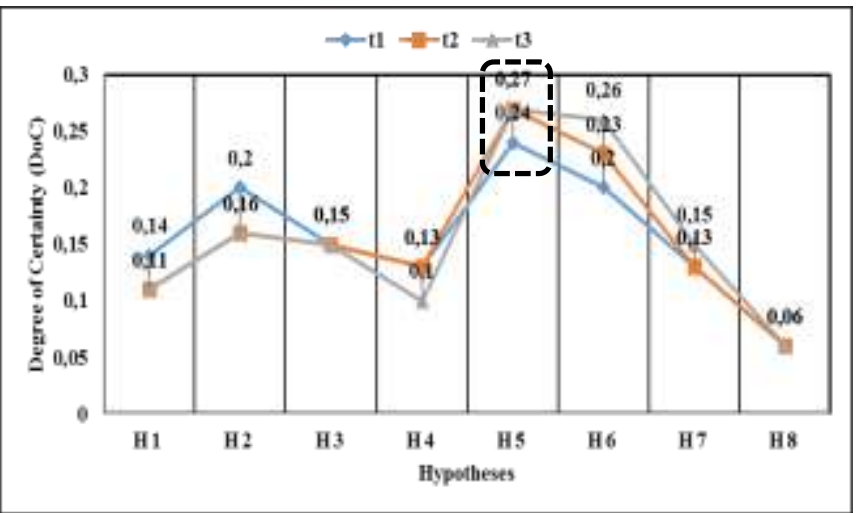

Fig. 4 The NKPDT of the system after three observation times.

Directly, we can compute the system's DoC after two more observation times, namely $t_{2}$ and $t_{3}$ by applying (3) as follows.

$$
\begin{aligned}
D o C_{t_{2}} & =\left|P\left(\theta_{5}\right)_{\text {estimate }}-\phi_{2}^{5}\right| \times 100 \% \\
& =|0.27-0| \times 100 \% \\
& =27 \% \\
D o C_{t_{3}} & =\left|P\left(\theta_{5}\right)_{\text {estimate }}-\phi_{3}^{5}\right| \times 100 \% \\
& =|0.27-0| \times 100 \% \\
& =27 \%
\end{aligned}
$$

From the three observation times as presented in Table II, the system is more confident that the sensed phenomenon is $H_{5}$. This finding shows two things as follow.

- We have proved that inferencing is carried out by fusing information from a pattern of sensed phenomenon with knowledge already stored by the system.

- We also have proved that obtaining an inference has to be done through information fusion where the inference is new knowledge of the system. This process can be said as knowlegde extraction, namely the creation of knowledge.

\section{v. Concluding Remarks}

If we generalize "inferencing" definition by replacing word "word" with "phenomenon", we can devise a new definition of knowledge extraction from AI perspective. But we have to redefine "inferencing" first. Our proposal for "inferencing" is the practice of inferring the meaning of an unfamiliar phenomenon from familiar phenomena occurring with it in a context together with system's knowledge of or beliefs about the world (environment). Therefore knowledge extraction would be the creation of new knowledge of unknown phenomenon sensed by a system by fusing the information from its sensor organ with its knowledge as the time passes. System in this context is an information processing system.

Having proved our technique along with a real-life example, we are happy to declare that A3S is a new method for knowledge extraction. The advantage of our method is it has facility to ensure that the knowledge it extracts from a pattern of sensed phenomenon. This facility is a mechanism to fuse the extracted knowledge from each observation time. This what we call as hierarchical knowledge extraction and this can be done by applying OMA3S method.

\section{References}

[1] A.D.W. Sumari, A.S. Ahmad, A.I. Wuryandari, and J. Sembiring, "Constructing brain-inspired knowledge-growing system: a review and a design concept", Proc. of Second Int. Con. on Distributed Framework and Applications 2010 (DFmA2010), 2-3 August 2010, pp. 95-102.

[2] "What's the difference between "inferencing" and "inferring"?". [Online]. Available: http://english.stackexchange.com/questions/123403/ whats-the-difference-between-inferencing-and-inferring.

[3] "1: Historical Perspectives on Alternative Assessment". [Online]. Available: http://textbooks.brookespublishing.com/losardo/chapter1/ keyterms.htm.

[4] "information processing". [Online]. Available: http://global.britannica.com/EBchecked/topic/287847/informationprocessing.

[5] "Wickens' Model". [Online]. Available: https://www.hf.faa.gov/ webtraining/Cognition/CogFinal008.htm.

[6] A.T. Welford, Skilled Performance: Perceptual and Motor Skills. Palo Alto, CA: Scott, Foresman and Company, 1976 in "Decision-making and Motor Behavior". [Online]. Available: http://www.kin.sjsu.edu/faculty/ewughalter/UG\%20ML\%20Decisionmaking\%20and\%20Motor\%20Behavior.ppt.

[7] "Models of information processing". [Online]. Available: https://sites.google.com/site/jacksrevisionwebsite/pe-revision/as-pe/aspe-theoretical-models/whiting-s-model.

[8] P. Ceravolo, M. C. Nocerino, and M. Viviani, "Knowledge Extraction from Semi-structured Data Based on Fuzzy Techniques" in M.G h. Negoita et al. (Eds.): KES 2004, LNAI 3215, pp. 328-334, 2004, Springer-Verlag Berlin.

[9] W. Dakka and P. G. Ipeirotis, "Automatic Extraction of Useful Facet Hierarchies from Text Databases". [Online]. Available: http://www4.ncsu.edu/ mbcusick/papers/dakka2008automatic.pdf.

[10] V. Presutti, A. G. Nuzzolese, S. Consoli, D. R. Recupero and A. Gangemi, "From Hyperlinks to Semantic Web Properties using Open Knowledge Extraction". [Online]. Available: http://www.semantic-webjournal.net/system/files/swj908.pdf.

[11] S. Costantini, N. Florio, and A. Paolucci, "A framework for structured knowledge extraction and representation from natural language via deep sentence analysis". [Online]. Available: http://ceur-ws.org/Vol810/paper-118.pdf.

[12] M. Vargas-Vera, E. Motta, J. Domingue, S. B. Shum and M. Lanzoni, "Knowledge Extraction by using an Ontologybased Annotation Tool". [Online]. Available: http://eecs.ceas.uc.edu/ mazlack/ ECE.716.Sp2011/Semantic.Web.Ontology.Papers/vargas.vera.2001.pdf. 
Proc. of the Fourth International Conference on Advances in Computing, Electronics and Communication - ACEC 2016. Copyright (C) Institute of Research Engineers and Doctors. All rights reserved.

ISBN: 978-1-63248-113-9 doi: 10.15224/ 978-1-63248-113-9-05

[13] A.D.W. Sumari, "The modeling of knowledge growing system based on multi-time observation A3S (OMA3S)", Tech. Rep., Institut Teknologi Bandung, 2009.

[14] A.D.W. Sumari, A.S. Ahmad, A.I. Wuryandari dan J. Sembiring, A Mathematical Model of Knowledge-Growing System: A Novel Perspective in Artificial Intelligence, Proc. of IndoMS Int. Conf. on Mathematics and Its Applications 2009 (IICMA2009), Gadjah Mada University, 12-13 October 2009, pp. 229-240.

[15] A.D.W. Sumari, "The modeling of Knowledge-Growing System based on Observation Multi-time Arwin-Adang-Aciek-Sembiring (OMA3S) method," EL-8095 Technical Report, School of Electrical Engineering and Informatics, Institut Teknologi Bandung, May 2009.

About Author (s):

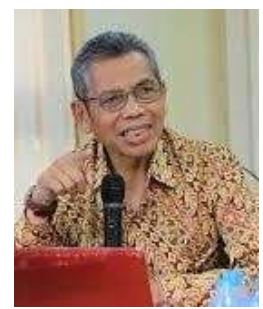

Adang Suwandi Ahmad (ASA) received his engineering degree (Ir.) in Electrical Engineering from ITB in 1976, Diplome Etude Approfondi Signaux et Bruits (DEA) option Electronique, and Docteur Ingenieur Signaux et Bruits option Electronique (Dr.-ing) from Universite des Sciences et Techniques du Languedoc Montpellier, France in Sept 1978 and August 1980 respectively. He became Institut Teknologi Bandung's Professor in Intelligent Electronics Instrumentation System in 2000. ASA's past researches were in Electronics Instrumentation Systems (Devices and Systems) and Intelligent Electronics Systems/Artificial Intelligence. Cooperation with Navy R\&D Service (1992) had yielded a War Game Simulator. In. $2002-2008$, He was the head of C4I (TNI PUSDALOPS) development team ITB-TNI. He founded Intelligent System Research Group(ISRG)ITB in1993 and since 2014 became Cognitive Artificial Intelligent Reseach Group (CAIRG) ITB. Now he focuses in bioinformatics computation, and Cognitive Artificial Intelligent-based instrumentation systems. He is the former Dean $(2006-2010)$ of the School of Electrical Engineering and Informatics ITB, Bandung, Indonesia. Currently, Prof. Ahmad is still with School of Electrical Engineering and Informatics ITB for some important researches in AI field especially in Cognitive Artificial Intelligent System area. He is currently using Information Fusion / KGS on some intelligence information for National Defence System.

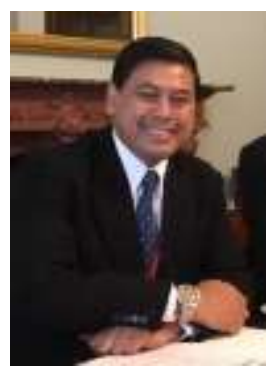

Colonel (Electronics) Arwin Datumaya "Daemon" Wahyudi Sumari was inaugurated as 2nd Lieutenant Officer of Electronics Corps of the Indonesian Air Force from Indonesian Air Force Academy with Honors in 1991. He also achieved Adi Makayasa Medal as Best of the Best Graduate of IDAFA and Trophy for the Best Graduate of 1991 Electronics Majoring. He received Sarjana Teknik (S.T.) degree in Electronics Engineering, Magister Teknik (M.T.) degree in Computer Engineering, and Doctor (Dr.) in Electrical Engineering and Informatics from Institut Teknologi Bandung (ITB), Bandung, Indonesia in April 1996, March 2008, and July 2010. All academics degree were achieved with Cum Laude predicate. His research interests include Artificial Intelligence, knowledge growing based system, cognitive science, and avionics system.. He has written more than 120 technical papers published in international and national books, journal, and proceedings as well as technical magazines. Col. Sumari is the first IDAF officer who received Certificate of Achievement from Chief of Staff of the Indonesian Air Force in 1996, the First Winner of Indonesian Armed Force's Research and Development Competition in 2001, and achieved the Best Manuscript Award "Sastratma" when studying at Indonesian Air Force's School of Staff and Command in 2011. He is now Policy Analyst at Secretariat General of National Resilience Council of the Republic of Indonesia. Beside his primary assignment, he is also a Senior Researcher at Cognitive Artificial Intelligence Research Group (CAIRG), School of Electrical Engineering and Informatics, Institut Teknologi Bandung, and Head of Information Resilience Division,
Desk for National Cyberspace, Coordinating Ministry for Political, Legal and Security Affairs. 